\title{
Molecular Biomarkers Associated with Early-Onset Symptomatic Intracranial Atherosclerosis
}

\author{
Yunfang Luo ${ }^{\mathrm{l}, *}$ \\ Fang $\mathrm{Yu}^{\mathrm{l}, *}$ \\ Xianjing Feng ${ }^{\prime}$ \\ Di Liao' \\ Yuanlin Ying' \\ Xi Li $\mathbb{D}^{\prime}$ \\ Qin Huang' \\ Zeyu Liu' \\ Minping Wei ${ }^{1}$ \\ Tingting Zhao' \\ Lin Zhang' \\ Ruxin Tu (D) \\ Jian Xia ${ }^{1-3}$ \\ 'Department of Neurology, Xiangya \\ Hospital, Central South University, \\ Changsha, Hunan, People's Republic of \\ China; ${ }^{2}$ Clinical Research Center for \\ Cerebrovascular Disease of Hunan \\ Province, Central South University, \\ Changsha, Hunan, People's Republic of \\ China; ${ }^{3}$ National Clinical Research \\ Center for Geriatric Disorders, Xiangya \\ Hospital, Central South University, \\ Changsha, Hunan, People's Republic of \\ China
}

*These authors contributed equally to this work
Correspondence: Jian Xia

Department of Neurology, Xiangya

Hospital, Central South University,

Changsha, Hunan, 4I0008, People's

Republic of China

Tel +86 73I 84327216

Fax +8673184327401 .

Email xjian1216@csu.edu.cn
Purpose: Previous studies have shown a rising incidence of early-onset symptomatic intracranial atherosclerosis (sICAS), which has brought a severe economic burden to social development. This study aimed to evaluate the molecular biomarkers associated with earlyonset sICAS and to seek possible intervention strategies for early prevention.

Patients and Methods: We consecutively recruited patients with sICAS and divided them into two groups based on age: early-onset sICAS group as age $\leq 60$ years old and late-onset sICAS group as age $>60$ years old. We collected and compared the demographic data and laboratory results of each group. A bivariate logistic regression model was applied to evaluate the independent molecular biomarkers of early-onset sICAS.

Results: A total of 1007 subjects with sICAS were enrolled in this study, comprising 519 patients in the early-onset sICAS group and 488 patients in the late-onset sICAS group. Bivariate logistic regression analysis demonstrated an increased level of white blood cell, platelet, albumin globulin ratio, free triiodothyronine, and a decreased level of total bile acid, urea nitrogen, high-density lipoprotein, homocysteine, and fibrinogen in the early-onset sICAS group when compared to the late-onset group.

Conclusion: Our study showed the relevance between early sICAS and circulating levels of different molecular biomarkers. Detection of these related molecular biomarkers may provide a simple way for early sICAS preventions in the future.

Keywords: ischemic stroke, symptomatic intracranial atherosclerosis, molecular biomarkers

\section{Introduction}

Intracranial atherosclerosis (ICAS) is the leading cause of ischemic stroke around the world. ICAS could be symptomatic or asymptomatic, and the symptomatic intracranial atherosclerosis (sICAS) refers to the ischemic stroke or transient ischemic attack (TIA) that occurs in the stenosis area of the supplying artery. ${ }^{1}$ sICAS is associated with higher rates of severe symptoms, recurrence and rehospitalization. ${ }^{2-5}$ A recent study showed that $45 \%-62 \%$ of patients with ischemic stroke had intracranial plaque and stenosis. ${ }^{6}$ Generally, age is an independent risk factor for sICAS, and the incidence of sICAS increases in the elderly. ${ }^{7}$ However, the occurrence of sICAS has shown a younger trend in recent years. Several studies have shown that risk factors are more complex and patients are more prone to worse prognosis in early-onset sICAS than in late-onset sICAS. ${ }^{8-10}$ Early-onset sICAS affects patients' quality of life and ability to work, and thus brings a heavy economic burden to the family and society.

In the past two decades, several researchers have sought to determine biomarkers correlated with sICAS, such as adipokines, interleukin-6, C-reactive protein, and 
matrix metalloproteinases. ${ }^{11}$ What's more, laboratory indicators including white blood cells, platelets, albumin/globulin ratio, and free triiodothyronine are considered to be independent risk factors for intracranial atherosclerotic diseases. $^{12-14}$ The interaction among white blood cells, platelets and endothelial cells is the leading cause of inflammation and atherosclerosis. ${ }^{15}$ However, serum albumin can attenuate the oxidative damage of atherosclerotic lesions. ${ }^{16}$ High level of preoperative serum-free thyroxine 4 can be used as a sensitive and independent predictor of vascular restenosis in patients with angina pectoris after bare stent implantation. ${ }^{17}$ To date, little is known about the prevalence and risk factors of early-onset sICAS. Genetic risk factors like RNF213 p.R4810K variant and RNF213 $\operatorname{cg} 22443212$ hypermethylation are reported to be associated with early-onset sICAS. ${ }^{18,19}$ However, circulating biomarkers of early-onset ICAS have not been widely explored.

In the present study, we aimed to explore molecular biomarkers associated with early-onset sICAS and seek an intervention tactic for earlier preventions against these factors in the future.

\section{Patients and Methods}

\section{Study Population}

The flow chart of this study is shown in Figure 1. We consecutively recruited 1007 sICAS patients in Xiangya Hospital of Central South University from August 2012 to May 2019. The diagnosis of ischemic stroke or TIA conforms to the Chinese guidelines for the diagnosis and treatment of acute ischemic stroke in $2018 .^{20}$ All patients were classified into two groups based on their age: early-onset group defined as age $\leq 60$ years old, and a late-onset group as age $>60$ years old. The inclusion criteria were as follows: 1. The age between 18 and 80 years old and the time from symptomonset to hospital $\leq 14$ days; 2 . All the subjects met the criteria for clinical diagnosis of ischemic stroke and transient ischemic attack. We excluded patients with the following clinical characteristics: 1 . Patients with cardiogenic stroke or other non-atherosclerotic causes diseases; 2. Imaging examinations indicated no intracranial artery stenosis or extracranial artery stenosis; 3. Other intracranial artery stenosis causes, such as moyamoya disease and vasculitis; 4. The patients have recent diseases, such as infection, tumor and autoimmune rheumatism; 5. Severe liver and kidney

Time from symptom-onset

to hospital $\leq 14$ days

Excluded:

- other non-atherosclerotic diseases $(n=720)$

- extracranial artery stenosis $(n=234)$

- moyamoya disease $(n=76)$

- vasculitis $(n=82)$

- infection or tumor $(n=185)$

- autoimmune rheumatism ( $n=97)$

- severe liver and kidney insufficiency or heart and respiratory failure $(n=123)$

- lacking laboratory date or imaging examinations $(n=358)$

- no signed consent forms $(n=276)$

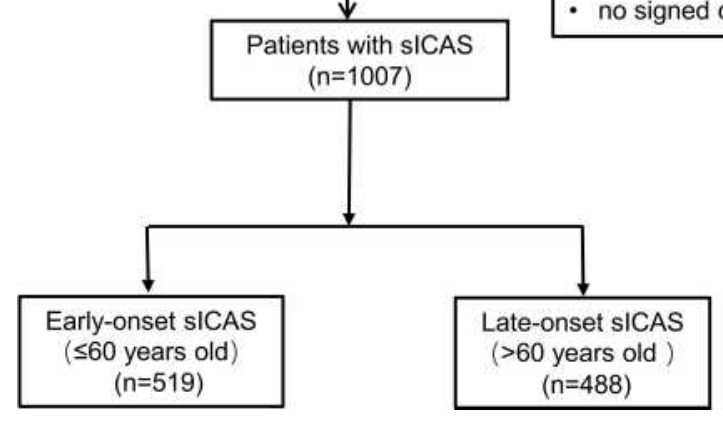

Figure I Patient selection flowchart.

Abbreviations: sICAS, symptomatic intracranial atherosclerosis; TIA, transient ischemic attacks. 
insufficiency or heart and respiratory failure; 6. Lacking data of laboratory or imaging examinations; 7 . None of the signed consent forms. Written informed consent was obtained from each patient included in the study, the study protocol conforms to the ethical guidelines of the 1975 Declaration of Helsinki and was approved by the Ethics Committee of Xiangya Hospital of Central South University, China.

\section{Clinical Data Collection}

Demographic information included age, sex, stroke history, family history of stroke, vascular risk factors (diabetes mellitus, hypertension, coronary artery disease, dyslipidemia, Table 1), alcohol intake, and cigarette smoking. The patient's peripheral venous blood was drawn in the next morning after admission, and was immediately examined in the Biochemical Laboratory of Xiangya Hospital. We recorded the plasma levels of blood routine, hepatic and renal function, plasma glucose and lipids, blood coagulation, thyroid function, and homocysteine. Most subjects underwent routine MRI examinations in a $3.0 \mathrm{~T}$ or $1.5 \mathrm{~T}$ MRI scanner, which composed of three-dimensional time-of-flight magnetic resonance angiography (3D-TOF-MRA), contrastenhanced magnetic resonance angiography (CE-MRA), T2/T1-weighted magnetic resonance images and fluid attenuated inversion recovery (FLAIR). Computed tomography (CT) and computed tomography angiography (CTA) were used to supplement those who cannot complete or cooperate with MRI. All patients had completed the carotid Doppler angiography. The DSA (gold standard), 3D-TOF-MRA and CTA were performed to distinguish the location and severity of intracranial artery stenosis. The intracranial artery stenosis was determined according to the Warfarin-Aspirin Symptomatic Intracranial Disease (WASID) Study: percent stenosis = $\left[1-\left(D_{\text {stenosis }} / D_{\text {normal }}\right)\right] \times 100 \%\left(D_{\text {stenosis }}\right.$ : the diameter of the vessel at the site of stenosis; $D_{\text {normal }}$ : the diameter of the normal vessel just distal to the stenosis). ${ }^{21}$ The severity of stenosis was divided into four grades: 1 . mild stenosis: $<50 \%$; 2 . moderate stenosis: $50 \%$ to $69 \%$; 3 . severe stenosis: $70 \%$ to $99 \%$; 4 . occlusion: no blood flow. The National Institutes of Health Stroke Scale (NIHSS) score was independently evaluated by two experienced clinicians at admission to evaluate the severity of stroke.

\section{Definition of Vascular Indicators}

Hypertension was diagnosed when systolic blood pressure $\geq 140 \mathrm{mmHg}$ and/or diastolic blood pressure $\geq 90 \mathrm{mmHg}$ or undergoing treatment of antihypertensive medications. Diabetes was diagnosed when fasting blood glucose (FBG) greater than $7 \mathrm{mmol} / \mathrm{L}$ and/or postprandial glucose (PPG) greater than $11.1 \mathrm{mmol} / \mathrm{L}$ or intake of antidiabetic drugs. Dyslipidemia was defined as total cholesterol (TC) $\geq 5.18 \mathrm{mmol} / \mathrm{L}$ or triglyceride $(\mathrm{TG}) \geq 1.70 \mathrm{mmol} / \mathrm{L}$ or lowdensity lipoprotein (LDL) $>3.19 \mathrm{mmol} / \mathrm{L}$ or high-density lipoprotein HDL $<1.04 \mathrm{mmol} / \mathrm{L}$. Stroke history referred to the patients who had incurred a single event of ischemic stroke or transient ischemic attack. Smokers were considered as the subject who had smoked continuously for 1 year with $\geq 1$ cigarette per day. Alcohol consumption was defined as average drinking greater than 2 standards for males or 1 standard for females per day.

\section{Statistical Methods}

All statistics were carried out using SPSS 22.0 software (IBM). We denoted categorical variables with absolute values and percentages. Continuous variables were presented as means \pm standard deviations ( $\pm \mathrm{SD}$ ) when conformed to the normal distribution or median (interquartile range (IQR)) when the data were non-normally distributed. To analyze the differences between groups, a chi-square test $\left(\chi^{2}\right)$ was performed on categorical covariates, while a Student's $t$-test or Mann-Whitney $U$-test and one-way ANOVA or KruskalWallis test were performed on continuous data. We used the median to classify the continuous variables into lower and higher groups in the bivariate correlation analysis. In the logistic regression analysis, variables with p-values $<0.05$ in the univariate analyses were included. In the overall statistical analysis, p-values $<0.05$ were considered statistically significant.

\section{Results}

\section{Baseline Characteristics of All Patients}

From August 2012 to May 2019, a total of 1007 patients confirmed with sICAS were recruited, 488 patients had early-onset sICAS and 519 patients had late-onset sICAS. The median age was 61 years old (IQR: 52-68 years old), including 645 males (64.1\%) and 362 females $(35.9 \%)$, with a male-to-female ratio of $1.78: 1$. The median NIHSS score at admission was 4 (IQR: 2-8). Other demographic data and risk factors are shown in Table 1, and the most common cardiovascular risk factor was hypertension (76.4\%), followed by smoking (44.1\%) and diabetes (33.8\%). 
Table I Baseline Characteristics of sICAS Patients

\begin{tabular}{|c|c|}
\hline Variables & Patients $(n=1007)$ \\
\hline Age (years) (median, IQR) & $61(52-68)$ \\
\hline Sex (male, N, \%) & $645(64.1 \%)$ \\
\hline Smoking (N, \%) & 444 (44.1\%) \\
\hline Drinking (N, \%) & $334(33.2 \%)$ \\
\hline \multicolumn{2}{|l|}{ History } \\
\hline Stroke History (N, \%) & $204(20.3 \%)$ \\
\hline Hypertension (N, \%) & 769 (76.4\%) \\
\hline $\mathrm{DM}(\mathrm{N}, \%)$ & $340(33.8 \%)$ \\
\hline Dyslipidemia (N, \%) & 477 (47.4\%) \\
\hline CAD (N, \%) & I50 (14.9\%) \\
\hline Family History of Stroke (N, \%) & $5 \mathrm{I}(5.1 \%)$ \\
\hline \multicolumn{2}{|l|}{ Ischemic type } \\
\hline Stroke (N, \%) & 954 (94.7\%) \\
\hline TIA (N, \%) & $53(5.3 \%)$ \\
\hline WBC $\left(\times 10^{9} / \mathrm{L}\right)$ (median, IQR) & $6.8(5.6-8.3)$ \\
\hline PLT $\left(\times 10^{9} / L\right)$ (median, IQR) & $204.0(166.0-248.0)$ \\
\hline NLR (median, IQR) & $2.7(1.9-3.8)$ \\
\hline AGR (median, IQR) & $1.5(1.3-1.6)$ \\
\hline TBIL $(\mu \mathrm{mol} / \mathrm{L})$ (median, IQR) & $9.9(7.5-13.1)$ \\
\hline TBA $(\mu \mathrm{mol} / \mathrm{L})$ (median, IQR) & $3.5(2.1-5.9)$ \\
\hline BUN (mmol/L) (median, IQR) & $4.9(4.0-6.1)$ \\
\hline $\mathrm{Cr}(\mu \mathrm{mol} / \mathrm{L})($ median, IQR) & $83.0(71.2-97.1)$ \\
\hline UA ( $\mu \mathrm{mol} / \mathrm{L})$ (median, IQR) & $315.0(259.6-379.6)$ \\
\hline TG (mmol/L) (median, IQR) & $1.5(1.2-2.1)$ \\
\hline TC (mmol/L) (median, IQR) & $4.4(3.6-5.2)$ \\
\hline HDLC (mmol/L) (median, IQR) & $1.0(0.9-1.2)$ \\
\hline LDLC (mmol/L) (median, IQR) & $2.7(2.1-3.3)$ \\
\hline Hcy $(\mu \mathrm{mol} / L)$ (median, IQR) & $13.2(11.1-16.5)$ \\
\hline Fib (g/L) (median, IQR) & $3.3(2.8-4.0)$ \\
\hline TSH (ulU/mL) (median, IQR) & $1.8(1.2-3.0)$ \\
\hline FT3 (pmol/L) (median, IQR) & $4.0(3.5-4.4)$ \\
\hline FT4 (pmol/L) (median, IQR) & $15.4(\mid 3.5-17.6)$ \\
\hline NIHSS score at admission (median, IQR) & $4(2-8)$ \\
\hline
\end{tabular}

Abbreviations: sICAS, symptomatic intracranial atherosclerosis; DM, diabetes mellitus; CAD, coronary artery disease; TIA, transient ischemic attacks; WBC, white blood cell; PLT, platelet; NLR, neutrophil lymphocyte ratio; AGR, albumin/globulin ratio; TBIL, total bilirubin; TBA, total bile acid; BUN, blood urea nitrogen; $\mathrm{Cr}$, creatinine; UA, uric acid; TG, triglyceride; TC, total cholesterol; HDLC, high-density lipoprotein cholesterol; LDLC, low-density lipoprotein cholesterol; Hcy, homocysteine; Fib, fibrinogen; TSH, thyroidstimulating hormone; FT3, free triiodothyronine; FT4, free thyroxine 4; NIHSS, the National Institutes of Health Stroke Scale.

\section{Different Stenosis Characteristics Between Early-Onset and Late-Onset sICAS Groups}

We presented the discrepancy of stenosis distribution in Figure 2. The location of the stenosis between the two groups showed no significant difference neither in the anterior nor in the posterior cerebral circulation $(\mathrm{p}=0.257)$. Stenosis tends to be located in the anterior circulation of the two groups $(67.4 \%$ in the early-onset group vs $69.8 \%$ in the late-onset group). When compared with the late-onset group, the early-onset group had a lower number of stenosis, despite no apparent statistical significance between the two groups $(\mathrm{p}=0.693)$. Moreover, severe vascular stenosis was presented in the early-onset sICAS group when compared with the late-onset group (severe stenosis: $23.8 \%$ in the early-onset group vs $20.0 \%$ in the late-onset group; occlusion: $39.5 \%$ in the early-onset group vs $38.2 \%$ in the late-onset group; $\mathrm{p}=0.006$ ).

\section{Association Between Molecular Biomarkers and sICAS in Both Early-Onset and Late-Onset Group}

We performed a univariate analysis to compare risk factors and plasma molecular biomarkers between groups. As presented in Table 2, male, smoking, drinking, dyslipidemia and high levels of white blood cell (WBC), platelet (PLT), albumin globulin ratio (AGR), triglyceride (TG), free triiodothyronine (FT3) were more frequent in the early-onset group $(\mathrm{p}<0.05)$, while high frequency of hypertension, coronary artery disease (CAD), high levels of neutrophil lymphocyte ratio (NLR), total bile acid (TBA), blood urea nitrogen (BUN), high-density lipoprotein cholesterol (HDLC), homocysteine (Hcy) and fibrinogen (Fib) were found in the late-onset group $(p<0.05)$. No significant differences were found when referred to the history of stroke, diabetes, the levels of total bilirubin (TBIL), serum creatinine, total cholesterol, low-density lipoprotein and thyroid-stimulating hormone (TSH) between the two groups. The severity of stroke at admission was assessed using the National Institutes of Health Stroke Scale. We found a lower NIHSS score in the early-onset group (median, 4; IQR, 2-8) than in the late-onset group (median, 5; IQR, 2-8), while it showed no statistical difference after univariate analysis $(\mathrm{p}=0.062)$. 

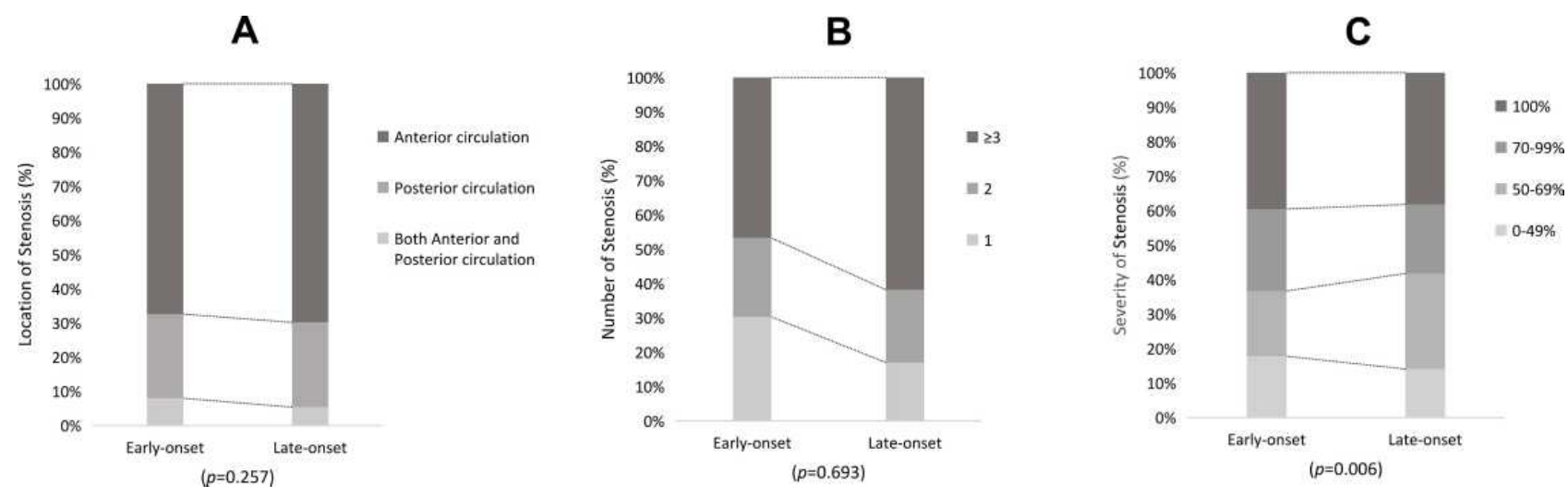

Figure 2 Different stenosis characteristics between early-onset and late-onset sICAS group (A-C).

Notes: Early-onset sICAS group defined as age $\leq 60$ years old and late-onset sICAS group defined as age $>60$ years old; P-values $<0.05$ were considered statistically significant.

\section{Logistic Regression Analyses Reveal the Independent Biomarkers Associated with the Early-Onset sICAS}

Statistically significant variables showing $p<0.05$ in the univariate analysis were selected as independent variables of the binary logistic regression analysis. As shown in Figure 3, a higher level of white blood cells (Adjusted OR, 95\% CI: 2.060 (1.360-3.120); $\mathrm{p}=0.001$ ), PLT (Adjusted OR, 95\% CI: 1.567 (1.046-2.348); $\mathrm{p}=0.029$ ), AGR (Adjusted OR, 95\% CI: $2.060(1.205-2.680) ; p=0.004)$ and FT3 (Adjusted OR, 95\% CI: 1.869 (1.259-2.775); $\mathrm{p}=0.002$ ), also a lower level of TBA (Adjusted OR, 95\% CI: 0.576, (0.391-0.849); $\mathrm{p}=0.005)$, BUN (Adjusted OR, 95\% CI: $0.573(0.390-0.840) ; \mathrm{p}=0.004)$, HDLC (Adjusted OR, 95\% CI: $0.621 \quad(0.417-0.926)$; $\mathrm{p}=0.020$ ), Hcy (Adjusted OR, 95\% CI: 0.485 (0.321-0.733); $\mathrm{p}=0.001$ ) and Fib (Adjusted OR, 95\% CI: 0.572 (0.381-0.859); $\mathrm{p}=0.007$ ) were independent risk factors of early-onset sICAS group after adjusting for the sex, stroke history, hypertension, coronary heart disease, diabetes, dyslipidemia, family history of stroke, smoking and drinking.

\section{Discussion}

Evidence focusing on biomarkers of early-onset sICAS is limited. In this study, we found that higher baseline levels of WBC, PLT, FT3, AGR and lower levels of TBA, BUN, HDLC, Hcy, and Fib were independent predictors of earlyonset sICAS.

Recent study has demonstrated that the WBC level in younger patients with the symptomatic intracranial atherosclerotic disease increases, ${ }^{22}$ which is in line with our study. As an inflammatory biomarker, WBC was associated with early atherosclerosis and late plaque formation in carotid artery diseases. ${ }^{23}$ WBC plays a crucial role in initiating and spreading atherosclerosis through rolling, adhesion, migration and activation. ${ }^{24}$ Activated WBCs tend to adhere to vascular endothelial cells and penetrate the intima, resulting in capillary leukocytosis and vascular resistance. ${ }^{25}$ Furthermore, WBCs release various hydrolases, cytokines and growth factors, leading to endothelial dysfunction, arterial elastic properties alteration and structural stiffness. ${ }^{25}$

Platelet also has an impact on the early stage of atherosclerotic injury. ${ }^{26,27}$ When the vascular injury occurs, the ruptured plaques initiate a series of responses involving platelet adhesion, platelet activators release, platelet morphological modification and subsequent granular contents release, and finally the glycoprotein IIb/IIIa receptors activation and the thrombus reaction enhancement. ${ }^{28}$ Various receptors markedly affect the firm platelet adhesion to the endothelial extracellular matrix, including glycoprotein receptors GPIb/IX/V, GPVI, GPIb, GPIIb/IIIa and collagen receptor a2b1. ${ }^{29}$ Activated platelets can further release a big heap of inflammatory mediators, inducing the chemotaxis, adhesion, and transmigration of the leukocytes to the inflammatory site, ${ }^{30}$ and promote the leukocyte recruitment and inflammatory response by morphology change, reactive oxygen species (ROS) production, neutrophil extracellular traps formation. ${ }^{23,31,32}$ Specific interactions with platelet-leukocyte may provide a therapeutic method for preventing early atherosclerotic lesions. ${ }^{14,24}$

The relationship between thyroid hormone and atherosclerosis is controversial. Some studies showed that thyroid hormones can stimulate atherosclerosis, while other studies indicated that thyroid hormones can inhibit atherosclerosis progression by regulating vasodilatation, 
Table 2 Comparison of Demographic, Laboratory and Clinical Features in Patients with Early and Late-Onset sICAS

\begin{tabular}{|c|c|c|c|}
\hline & $\begin{array}{l}\text { Early-Onset }(\leq 60) \\
n=488\end{array}$ & $\begin{array}{l}\text { Late-Onset }(>60) \\
n=519\end{array}$ & P-value \\
\hline Sex (male, N, \%) & $343(70.3 \%)$ & $302(58.2 \%)$ & $<0.001$ \\
\hline Smoking (N, \%) & $247(50.6 \%)$ & 197 (38.0\%) & $<0.001$ \\
\hline Drinking (N, \%) & 187 (38.3\%) & 147 (28.3\%) & 0.001 \\
\hline \multicolumn{4}{|l|}{ History } \\
\hline Stroke History (N, \%) & 95 (19.5\%) & $109(21.0 \%)$ & 0.545 \\
\hline Hypertension (N, \%) & 344 (70.5\%) & 425 (81.9\%) & $<0.001$ \\
\hline $\mathrm{DM}(\mathrm{N}, \%)$ & $168(34.4 \%)$ & $172(33.1 \%)$ & 0.666 \\
\hline Dyslipidemia (N, \%) & $258(52.9 \%)$ & $219(42.2 \%)$ & 0.001 \\
\hline CAD (N, \%) & $50(10.2 \%)$ & $100(19.3 \%)$ & $<0.001$ \\
\hline Family History of Stroke (N, \%) & $29(5.9 \%)$ & $22(4.2 \%)$ & 0.218 \\
\hline Ischemic type & & & 0.223 \\
\hline Stroke (N, \%) & $458(93.9 \%)$ & $496(95.6 \%)$ & \\
\hline TIA (N, \%) & $30(6.1 \%)$ & $23(4.4 \%)$ & \\
\hline WBC $\left(\times 10^{9} / \mathrm{L}\right)$ (median, IQR) & $7.0(5.7-8.5)$ & $6.5(5.4-8.0)$ & 0.002 \\
\hline PLT $\left(\times 10^{9} / L\right)$ (median, IQR) & $212.0(172.2-257.0)$ & $195.0(161.0-239.0)$ & $<0.001$ \\
\hline NLR (median, IQR) & $2.5(1.9-3.5)$ & $2.8(2.0-4.2)$ & 0.008 \\
\hline AGR (median, IQR) & $1.5(1.4-1.7)$ & $1.4(1.2-1.6)$ & $<0.001$ \\
\hline TBIL ( $\mu \mathrm{mol} / \mathrm{L})$ (median, IQR) & $9.9(7.2-12.6)$ & $9.9(7.6-13.7)$ & 0.181 \\
\hline TBA $(\mu \mathrm{mol} / \mathrm{L})($ median, IQR) & $3.1(2.0-4.9)$ & $3.8(2.4-6.6)$ & $<0.001$ \\
\hline BUN (mmol/L) (median, IQR) & $4.7(3.7-5.7)$ & $5.3(4.2-6.3)$ & $<0.001$ \\
\hline $\mathrm{Cr}(\mu \mathrm{mol} / \mathrm{L})($ median, IQR) & $82.1(71.0-94.0)$ & $83.0(71.7-98.0)$ & 0.194 \\
\hline UA ( $\mu$ mol/L) (median, IQR) & $316.6(264.0-37 \mid .6)$ & $310.8(254.5-386.6)$ & 0.607 \\
\hline TG (mmol/L) (median, IQR) & $1.7(1.2-2.3)$ & $1.4(1.1-1.9)$ & $<0.001$ \\
\hline TC (mmol/L) (median, IQR) & $4.3(3.5-5.2)$ & $4.4(3.6-5.2)$ & 0.188 \\
\hline HDLC (mmol/L) (median, IQR) & $1.0(0.8-1.2)$ & I.I $(0.9-1.3)$ & $<0.001$ \\
\hline LDLC (mmol/L) (median, IQR) & $2.6(2.1-3.3)$ & $2.7(2.1-3.3)$ & 0.595 \\
\hline Hcy $(\mu \mathrm{mol} / \mathrm{L})$ (median, IQR) & $12.6(10.7-15.2)$ & $13.7(\mid 1.3-17.6)$ & $<0.001$ \\
\hline Fib (g/L) (median, IQR) & $3.2(2.7-3.9)$ & $3.4(2.8-4.2)$ & 0.001 \\
\hline TSH (mlU/mL) (median, IQR) & $1.8(1.2-2.9)$ & $1.8(1.2-3.1)$ & 0.375 \\
\hline FT3 (pmol/L) (median, IQR) & $4.1(3.6-4.6)$ & $3.9(3.4-4.3)$ & $<0.001$ \\
\hline FT4 (pmol/L) (median, IQR) & $15.5(\mid 3.6-17.6)$ & $15.4(\mid 3.4-17.6)$ & 0.899 \\
\hline NIHSS score at admission (median, IQR) & $4(2-8)$ & $5(2-8)$ & 0.062 \\
\hline
\end{tabular}

Notes: P-values $<0.05$ were considered statistically significant.

macrophage function, and VSMC proliferation. ${ }^{33-35}$ Clinical evidence showed a correlation between the elevated FT3 level and the risk of subclinical and clinical atherosclerosis, ${ }^{36}$ indicating that increased thyroid hormones may lead to atherosclerosis. The role of FT3 in early-onset sICAS may involve the following three 


\begin{tabular}{|c|c|c|c|c|}
\hline & & & Adjusted Odds Ratio $(95 \% \mathrm{Cl})$ & P-value \\
\hline WBC $\geq 6.8 \times 10^{9} / \mathrm{L}$ & & 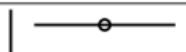 & $2.060(1.360-3.120)$ & 0.001 \\
\hline PLT $\geq 204 \times 10^{9} / \mathrm{L}$ & & Oـ & $1.567(1.046-2.348)$ & 0.029 \\
\hline$A G R \geq 1.5$ & & -0 & $1.797(1.205-2.680)$ & 0.004 \\
\hline $\mathrm{TBA} \geq 3.5 \mu \mathrm{mol} / \mathrm{L}$ & $\rightarrow$ & & $0.576(0.391-0.849)$ & 0.005 \\
\hline $\mathrm{BUN} \geq 4.9 \mathrm{mmol} / \mathrm{L}$ & $\rightarrow$ & & $0.573(0.390-0.840)$ & 0.004 \\
\hline $\mathrm{HDLC} \geq 1.0 \mathrm{mmol} / \mathrm{L}$ & - & & $0.621(0.417-0.926)$ & 0.020 \\
\hline $\mathrm{Hcy} \geq 13.2 \mu \mathrm{mol} / \mathrm{L}$ & $\theta$ & & $0.485(0.321-0.733)$ & 0.001 \\
\hline $\mathrm{Fib} \geq 3.3 \mathrm{~g} / \mathrm{L}$ & - & & $0.572(0.381-0.859)$ & 0.007 \\
\hline \multirow[t]{2}{*}{ FT3 $\geq 4.0 \mathrm{pmol} / \mathrm{L}$} & & 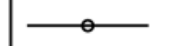 & $1.869(1.259-2.775)$ & 0.002 \\
\hline & 0 & 2 & & \\
\hline
\end{tabular}

Figure 3 Logistic regression analyses for factors independently associated with the early-onset sICAS.

Notes: The cut-off value for each biomarker was determined by the median. P-values $<0.05$ were considered statistically significant.

aspects: First, excessive thyroid hormone can accelerate the release of ROS, induce the adhesion to the endothelial cell, and lead to endothelial dysfunction. Second, thyroid hormone regulates the synthesis of procoagulant proteins and promotes plaque vulnerability and rupture. Third, thyroid hormone increases cardiac load and myocardial oxygen consumption, eventually leading to ischemic events and death. ${ }^{36}$ What's more, elevated level of antithyroid peroxidase antibody (TPO-Ab) was found in young (age $\leq 55$ years old) stroke patients with intracranial stenosis, ${ }^{37}$ and TPO-Ab could induce ICAS by promoting the migration of vascular smooth muscle cells. ${ }^{38}$ However, in our data, TPO-Ab level was excluded from the final analysis owing to a substantial number of missing values. The available data showed that patients in early-onset group had higher mean concentrations of TPO-Ab than those in the late group ( 44.92 vs $35.43 \mathrm{U} / \mathrm{mL}$ ). Further research should be conducted to confirm this issue.

In addition to the above-mentioned indicators, we found that the level of AGR was higher in the earlyonset group, that is, AGR was lower in late-onset sICAS patients. AGR refers to the ratio of plasma albumin to non-albumin protein, while non-albumin includes fibrinogen, prothrombin, inflammatory proteins and other regulatory proteins except globulin. ${ }^{39}$ Thus, not only the increased plasma albumin concentration but also the decreased fibrinogen level plays a crucial role in the high AGR ratio, which is consistent with our findings that lower fibrinogen level was found in the early-onset sICAS group. Clinical evidence has shown that low AGR has an adverse impact on atherosclerosis and cardiocerebrovascular events. ${ }^{39,40}$ Due to the increased aging associated with malnutrition and inflammatory reaction, albumin is decreasing in the elderly. ${ }^{39,40}$ High level of AGR might be just a biomarker of early-onset sICAS, while lower level of AGR and albumin may be risk factors for late-onset sICAS.

Besides, a reduced plasma level of TBA, BUN, HDLC, and Hcy was also found in the early-onset sICAS group. Previous studies have revealed that excessive cholesterol converts into bile acids, then discharges from feces in the form of bile salt, ${ }^{41}$ while HDLC transports cholesterol back to the liver from the outside in reverse transportation. ${ }^{42}$ All of the above results indicated that low levels of HDLC and TBA were risk factors for early sICAS. High level of BUN was considered to be associated with severe limb ischemia. ${ }^{43}$ Clinical evidence found that elevated Hcy accelerated atherosclerosis through monocyte activation, endothelial cell injury, lipid synthesis disorder, stimulation of VSMC proliferation and thrombosis. ${ }^{44}$ Although not well-understood for the lower levels of BUN and Hcy in the early-onset group than in the late-onset group, age may play a role leading to the decrease of these indicators. These findings indicate different pathophysiological mechanisms between early- and late-onset sICAS.

There are some limitations in our study. First, this study adopted a single-center clinical sample, and most patients lived in the central area of the Hunan province, resulting in a regional difference. Second, since it is a cross-sectional study, it is impossible to draw a causal inference between the early-onset sICAS and the risk factors. Third, the baseline plasma or serum biomarkers were collected at admission; however, it actually should be 
a dynamic process. Fourth, the number of TIA patients was relatively small, so we need to expand the sample size in the future. Fifth, tools for measuring ICAS were not unified with MRA/CE-MRA/CTA/DSA/, most patients were evaluated by MRA and CE-MRA, which may exaggerate the stenosis of intracranial arteries. Finally, earlyonset asymptomatic ICAS individuals were not included in this study, which is also of major importance.

Our research also has several strengths. First, this was a big clinical study with large sample size from Xiangya Hospital and included 1007 patients, which greatly improved the statistical confidence and reduced the random errors. Second, the molecular indicators obtained from the clinical serum samples of patients have the advantages of easy detection and low cost. Third, the mechanisms for early-onset intracranial atherosclerosis have not been reported previously, and there have been no clinical trials investigating the molecular index differences between the early and late-onset sICAS groups. Finally, our results suggest that the combined detection of these molecular indicators has a positive significance for identifying patients with a higher risk of early-onset sICAS, and close follow-up should be emphasized for these patients. The prognostic value and potential therapeutic effect of these molecular indicators are worth further study in the future.

\section{Conclusion}

In conclusion, high levels of WBC, Plt, AGR and FT3, as well as low levels of TBA, BUN, HDLC, Hcy and Fib are associated with early-onset sICAS when compared with late-onset sICAS. Detection of these molecular biomarkers may provide a simple way for the prevention and diagnosis of early sICAS. Identification of additional biomarkers associated with early-onset ICAS, either symptomatic or asymptomatic, should be discussed in the future.

\section{Ethics Approval and Informed Consent}

Written informed consent was obtained from each patient included in the study, the study protocol conforms to the ethical guidelines of the 1975 Declaration of Helsinki and has been priorly approved by the Ethics Committee of Xiangya Hospital of Central South University, China.

\section{Consent for Publication}

We confirm that the details of any images, videos, recordings, etc., can be published, and that the person(s) providing consent have been shown the article contents to be published.

\section{Acknowledgments}

We thank all patients for their participation in this study. We also wish to thank Dr. Changhan Chen from the Department of Otolaryngology Head and Neck Surgery, Xiangya Hospital, Central South University, for his help in language polishing.

\section{Author Contributions}

J.X. and F.Y. designed the study. Y.L. wrote the manuscript. F.Y. and Y.L. performed the analysis and interpretation of data as well as the manuscript revision. X.F., D.L., M.W., X.L., Q. H., Z.L., L.Z., T.Z., and R.T. were responsible for the acquisition and interpretation of data. All authors made a significant contribution to the work reported, whether that is in the conception, study design, execution, acquisition of data, analysis and interpretation; took part in drafting, revising or critically reviewing the article; gave final approval of the version to be published; have agreed on the journal to which the article has been submitted; and agree to be accountable for all aspects of the work.

\section{Funding}

This work was supported by the National Natural Science Foundation of China (Grant NO. 81671166), Provincial Key Plan for Research and Development of Hunan (Grant No. 2020SK2067), Project Program of National Clinical Research Center for Geriatric Disorders (Xiangya Hospital, Grant No. 2020LNJJ16), and the Fundamental Research Funds for the Central Universities of Central South University (Grant No. 2019zzts902).

\section{Disclosure}

The authors report no conflicts of interest in this work.

\section{References}

1. Ma YH, Leng XY, Dong Y, et al. Risk factors for intracranial atherosclerosis: a systematic review and meta-analysis. Atherosclerosis. 2019;281:71-77.

2. Wang Y, Zhao X, Liu L, et al. Prevalence and outcomes of symptomatic intracranial large artery stenoses and occlusions in China: the Chinese Intracranial Atherosclerosis (CICAS) Study. Stroke. 2014;45 (3):663-669. 
3. Tsivgoulis G, Vadikolias K, Heliopoulos I, et al. Prevalence of symptomatic intracranial atherosclerosis in Caucasians: a prospective, multicenter, transcranial Doppler study. J Neuroimaging. 2014;24(1):11-17.

4. Battistella V, Elkind M. Intracranial atherosclerotic disease. Eur j Neurol. 2014;21(7):956-962.

5. Massberg S, Brand K, Grüner S, et al. A critical role of platelet adhesion in the initiation of atherosclerotic lesion formation. $J \operatorname{Exp}$ Med. 2002;196(7):887-896.

6. Ritz K, Denswil NP, Stam OCG, et al. Cause and mechanisms of intracranial atherosclerosis. Circulation. 2014;130(16):1407-1414.

7. Park JH, Kwon HM, Roh JK. Metabolic syndrome is more associated with intracranial atherosclerosis than extracranial atherosclerosis. Eur J Neurol. 2007;14(4):379-386.

8. Celik A, Ozcetin M, Celikyay ZRY, et al. Evaluation of possible subclinical atherosclerosis in adolescents with a family history of premature atherosclerosis. Atherosclerosis. 2012;222(2):537-540.

9. Maaijwee NA, Rutten-Jacobs LC, Schaapsmeerders P, et al. Ischaemic stroke in young adults: risk factors and long-term consequences. Nat Rev Neurol. 2014;10(6):315-325.

10. Terni E, Giannini N, Brondi M, et al. Genetics of ischaemic stroke in young adults. BBA Clin. 2014;3:96-106.

11. Wang Y, Meng R, Liu G, et al. Intracranial atherosclerotic disease. Neurobiol Dis. 2019;124:118-132.

12. Kazmierski R, Guzik P, Ambrosius W, et al. Predictive value of white blood cell count on admission for in-hospital mortality in acute stroke patients. Clin Neurol Neurosurg. 2004;107(1):38-43.

13. Yildirim T, Akin F, Altun I, et al. Platelet indices and stroke. Ren Fail. 2018;40(1):265.

14. Wu MD, Atkinson TM, Lindner JR. Platelets and von Willebrand factor in atherogenesis. Blood. 2017;129(11):1415-1419.

15. Nagy B, Miszti-Blasius K, Kerenyi A, et al. Potential therapeutic targeting of platelet-mediated cellular interactions in atherosclerosis and inflammation. Curr Med Chem. 2012;19(4):518-531.

16. Lapenna D, Ciofani G, Ucchino S, et al. Serum albumin and biomolecular oxidative damage of human atherosclerotic plaques. Clin Biochem. 2010;43(18):1458-1460.

17. Canpolat U, Turak O, Özcan F, et al. Impact of free thyroxine levels and other clinical factors on bare metal stent restenosis. Arch Endocrinol Metab. 2017;61(2):130-136.

18. Kittner SJ, Singhal AB. Premature atherosclerosis: a major contributor to early-onset ischemic stroke. Neurology. 2013;80 (14):1272-1273.

19. Kamimura T, Okazaki S, Morimoto T, et al. Prevalence of RNF213 p. $\mathrm{R} 4810 \mathrm{~K}$ variant in early-onset stroke with intracranial arterial stenosis. Stroke. 2019;50(6):1561-1563.

20. Neurology C, Society C. Chinese guidelines for diagnosis and treatment of acute ischemic stroke 2018. Chinese J Neurol. 2018;51:666-682.

21. Barnett HJM, Taylor DW, Haynes RB, et al. Beneficial effect of carotid endarterectomy in symptomatic patients with high-grade carotid stenosis. N Engl J Med. 1991;325(7):445-453.

22. Vbiagele B, Lynn MJ, Saver JL, et al. Leukocyte count and vascular risk in symptomatic intracranial atherosclerosis. Cerebrovasc Dis. 2007;24(2-3):283-288.

23. Ortega E, Gilabert R, Nuñez I, et al. White blood cell count is associated with carotid and femoral atherosclerosis. Atherosclerosis. 2012;221(1):275-281.

24. Seizer P, May AE. Therapeutic potential and strategies against leukocyte-platelet interaction in atherosclerosis. Curr Vasc Pharmacol. 2012;10(5):550-554.
25. Sekitani Y, Hayashida N, Kadota K, et al. White blood cell count and cardiovascular biomarkers of atherosclerosis. Biomarkers. 2010;15 (5):454-460.

26. Aukrust P, Halvorsen B, Ueland T, et al. Activated platelets and atherosclerosis. Expert Rev Cardiovasc Ther. 2010;8(9):1297-1307.

27. Dotsenko O, Chaturvedi N, Thom SA, et al. Platelet and leukocyte activation, atherosclerosis and inflammation in European and South Asian men. J Thromb Haemost. 2007;5(10):2036-2042.

28. Davis KA, Miyares MA, Dietrich E. Dual antiplatelet therapy with clopidogrel and aspirin after ischemic stroke: a review of the evidence. Am J Health Syst Pharm. 2015;72(19):1623-1629.

29. Yeung J, Li W, Holinstat M. Platelet signaling and disease: targeted therapy for thrombosis and other related diseases. Pharmacol Rev. 2018;70(3):526-548.

30. Barrett TJ, Schlegel M, Zhou F. Platelet regulation of myeloid suppressor of cytokine signaling 3 accelerates atherosclerosis. Sci Transl Med. 2019;11:517.

31. Lindemann S, Krämer B, Seizer P, Gawaz M. Platelets, inflammation and atherosclerosis. J Thromb Haemost. 2007;5(Suppl 1):203-211.

32. Gerdes N, Seijkens T, Lievens D, et al. Platelet CD40 exacerbates atherosclerosis by transcellular activation of endothelial cells and leukocytes. Arterioscler Thromb Vasc Biol. 2016;36(3):482-490.

33. Fukuyama K, Ichiki T, Takeda K, et al. Downregulation of vascular angiotensin II type 1 receptor by thyroid hormone. Hypertension. 2003;41(3):598-603.

34. Perrotta C, Buldorini M, Assi E, et al. The thyroid hormone triiodothyronine controls macrophage maturation and functions: protective role during inflammation. Am J Pathol. 2014;184(1):230-247.

35. Cappola AR, Ladenson PW. Hypothyroidism and atherosclerosis. $J$ Clin Endocrinol Metab. 2003;88(6):2438-2444.

36. Bano A, Chaker L, Mattace-Raso FUS, et al. Thyroid function and the risk of atherosclerotic cardiovascular morbidity and mortality: the Rotterdam study. Circ Res. 2017;121(12):1392-1400.

37. Shi Z, Zhang X, Chen Z, et al. Elevated thyroid autoantibodies and intracranial stenosis in stroke at an early age. Int J Stroke. 2014;9 (6):735-740.

38. Zhang X, Zhou Y, Ding W, et al. TPO-Ab plays a role in arterial remodeling in patients with intracranial stenosis. Atherosclerosis. 2019;280:140-146.

39. Azab B, Bibawy J, Harris K. Value of albumin-globulin ratio as a predictor of all-cause mortality after non-ST elevation myocardial infarction. Angiology. 2013;64(2):137-145.

40. Zhang Y, Cliff WJ, Schoefl GI, Higgins G. Plasma protein insudation as an index of early coronary atherogenesis. Am J Pathol. 1993;143 (2):496-506.

41. Kuipers F, Stroeve JH, Caron S, Staels B. Bile acids, farnesoid $\mathrm{X}$ receptor, atherosclerosis and metabolic control. Curr Opin Lipidol. 2007;18(3):289-297.

42. Tsompanidi EM, Brinkmeier MS, Fotiadou EH, Giakoumi SM, Kypreos KE. HDL biogenesis and functions: role of HDL quality and quantity in atherosclerosis. Atherosclerosis. 2010;208(1):3-9.

43. Gary T, Pichler M, Schilcher G, et al. Elevated blood urea nitrogen is associated with critical limb ischemia in peripheral arterial disease patients. Medicine. 2015;94(24):e948.

44. Yang F, Tan HM, Wang H. Hyperhomocysteinemia and atherosclerosis. Sheng li Xue Bao. 2005;57(2):103-114. 


\section{Publish your work in this journal}

Clinical Interventions in Aging is an international, peer-reviewed journal focusing on evidence-based reports on the value or lack thereof of treatments intended to prevent or delay the onset of maladaptive correlates of aging in human beings. This journal is indexed on PubMed Central, MedLine, CAS, Scopus and the Elsevier
Bibliographic databases. The manuscript management system is completely online and includes a very quick and fair peer-review system, which is all easy to use. Visit http://www.dovepress.com/ testimonials.php to read real quotes from published authors. 